\title{
Abandoned pacing lead - a trap and a source of subsequent technical complications during transvenous lead extraction
}

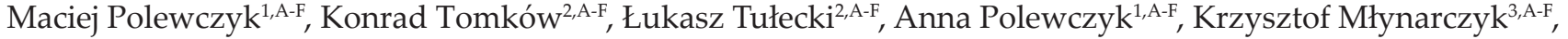 \\ Andrzej Kutarski ${ }^{4, A-F}$ \\ A - Research concept and design, B - Collection and/or assembly of data, C - Data analysis and interpretation, \\ D - Writing the article, E - Critical revision of the article, F - Final approval of article \\ 1. Institute of Medical Sciences, The Jan Kochanowski University, Kielce, Poland \\ 2. Department of Cardiac Surgery, The Pope John Paul II Province Hospital of Zamość Poland \\ 3. Department of Cardiology, Specialistic Hospital Tarnow, Poland \\ 4. Department of Cardiology, Medical University of Lublin, Poland
}

Address for correspondence:

Maciej Polewczyk, Institute of Medical Sciences, The Jan Kochanowski University, Kielce, Poland email: maciek.polewczyk@gmail.com

Konrad Tomków, Department of Cardiac Surgery, The Pope John Paul II Province Hospital of Zamość Poland email: konradtomkow@wp.pl

Łukasz Tułecki, Department of Cardiac Surgery, The Pope John Paul II Province Hospital of Zamość Poland
email: luke27@poczta.onet.pl

Anna Polewczyk, Institute of Medical Sciences, The Jan Kochanowski University, Kielce, Poland

email: AnnaPolewczyk@wp.pl

Krzysztof Młynarczyk, Department of Cardiology, Specialistic Hospital Tarnow, Poland

email: mlykrzy@wp.pl

Andrzej Kutarski, Department of Cardiology, Medical University of Lublin, Poland

email: a_kutarski@yahoo.com

Received: 2018-12-16

Revised:

Accepted: 2018-12-21

Final review: 2018-12-21

DOI: $10.24255 / \mathrm{hbj} / 102273$

Key words:

lead-to-lead adherence, transvenous lead extraction, lead-related infective endocarditis

\section{Abstract}

\section{Background}

We present a unique case of transvenous lead extraction with technical problems. Our experienced operator was able

A 77-year-old woman with a dual chamber pacemaker was admitted to the transvenous lead extraction (TLE) reference centre with suspected lead-related infective endocarditis (LRIE).

The pacemaker was implanted in 1998, and an additional lead was implanted in 2006 (during generator replacement) due to ventricular lead dysfunction. The last generator replacement was in 2016. During the current hospitalisation (2018), the transoesophageal echocardiography (TOE) revealed vegetations connected with the leads in the right atrium. Blood

to use strong lead-to-lead adherence to remove a broken lead without major or minor complications.

tests revealed highly elevated C-reactive protein (CRP) and procalcitonin levels. The blood cultures were positive for methicillin-sensitive Staphylococcus aureus (MSSA). The patient was diagnosed with LRIE and qualified for transvenous lead extraction. The procedure was performed in a hybrid operating room, under general anaesthesia with intraoperative TOE and with on-site cardiosurgical backup. As the patient was pacemaker-dependent, temporary pacing via femoral access was used. The leads were extracted using Byrd dilator sheaths (Cook, USA). At first, the bipolar ventricular lead (2006) was 
extracted without major difficulties. During further stages, the old (1998) unipolar (UP) ventricular lead was broken during extraction in the subclavian vein. At this point we decided to start the extraction of the atrial lead. During the extraction, we were surprised to find that the broken unipolar lead had moved into the Byrd dilator sheath together with the atrial lead due to strong lead-to-lead adherence. As part of the UP lead was removed with the bipolar atrial lead, the remaining fragment (still inside the dilator sheath) was successfully caught with a lasso catheter and extracted without major difficulties (Figure 1). The temporary pacing lead was intro- sheath, and dislodgement of a functional lead are the most common technical problems during TLE, which significantly prolong the procedure and unsolved properly may lead to severe procedural complications ${ }^{[1]}$. In our case, the experienced operator was able to transform a technical problem (strong mutual lead connection) into a method of solving another technical issue (lead break), which is extraordinary. A broken lead grasp with a lasso introduced by the Byrd dilator sheath was described earlier ${ }^{[2]}$. However, grasping a broken lead via recaptured subclavian access and the extraction of two leads using the same dilator sheath in our case was unusual.

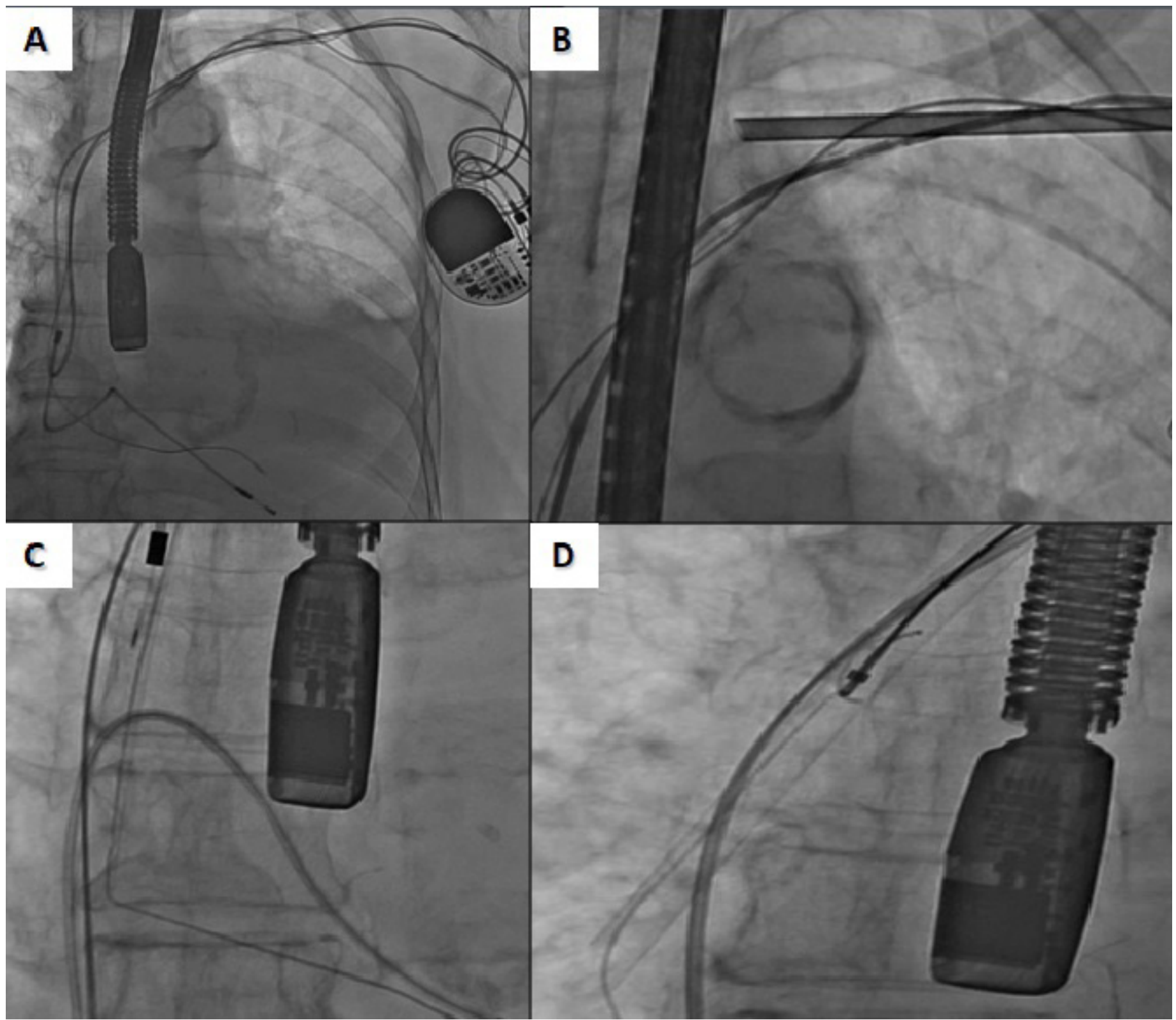

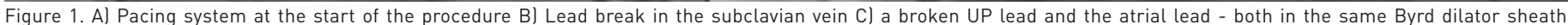
D) remaining lead fragment caught with lasso.

duced via the left subclavian approach, and the femoral one was removed. There were no periprocedural complications. After 4 weeks the patient received a new pacing system on the right side of the chest.

Strong lead-to-lead adherence, a lead break, a significant block in subclavian venous entry, damage of the polypropylene
An excessively long lead dwelling time is a known risk factor for developing dangerous complications during $\mathrm{TLE}^{[3]}$. In our case, the procedural risk could have been minimised if the 20-year-old abandoned lead had been extracted earlier. According to the current guidelines it is important to assess the potential risk and benefits for the patient during qualification 
for TLE of a (non-infected) abandoned lead ${ }^{[4]}$. Experts also underline the need to consider extraction of a non-functional lead in a patient with a long-life prognosis, which may prevent more risky and complex procedures in the future.

\section{References}

1. Kutarski A, Czajkowski M, Pietura R et al. Effectiveness, safety, and long-term outcomes of non-powered mechanical sheaths for transvenous lead extraction. Europace. 2017 Aug [Epub ahead of print]

2. Kutarski A, Pietura R, Czajkowski M. Breakage of extracted leads: another management option. Kardiol Pol. 2012; 70: 307-312.

3. Auricchio A, Regoli F, Conte G et al. Key lessons from the ELECTRa registry in the modern era of transvenous lead extraction. Arrhythm Electrophysiol Rev. 2017; 6: 111-113.

4. Kusumoto FM, Schoenfeld MH, Wilkoff BL. 2017 HRS expert consensus statement on cardiovascular implantable electronic device lead management and extraction. Heart Rhythm. 2017; 14: e503-e551. 